\title{
Towards Understanding Smartphone Usage and Sleep with a Crowdsensing Approach
}

\author{
Parsa Sharmila ${ }^{1}$, Vappu Schroderus ${ }^{1}$, Eemil Lagerspetz ${ }^{2}$, Ella Peltonen $^{1}$ \\ (1) Center for Ubiquitous Computing, University of Oulu, Finland \\ (2) Department of Computer Science, University of Helsinki, Finland \\ parsa.sharmila@oulu.fi,vappu.schroderus@student.oulu.fi,eemil.lagerspetz@helsinki.fi,ella.peltonen@oulu.fi
}

\begin{abstract}
Smartphone usage and sleep quality have established connections in psychological research, but in the HCI context, the topic is still understudied. In this paper, we present preliminary insights into behavioral patterns between smartphone usage and sleep quality by using crowdsensed data. We utilize a large-scale mobile usage dataset and a PHQ-8 depression questionnaire answered by 743 participants from varying age groups and socioeconomic backgrounds. Based on our preliminary results, we provide a methodological pipeline for future work towards understanding the relationship between daily smartphone usage patterns and sleep quality in the wild.
\end{abstract}

\section{CCS CONCEPTS}

- Human-centered computing $\rightarrow$ Smartphones; • Applied computing $\rightarrow$ Health informatics.

\section{KEYWORDS}

Smartphones; Sleep; Crowdsensing

\section{ACM Reference Format:}

Parsa Sharmila, Vappu Schroderus, Eemil Lagerspetz, Ella Peltonen. 2020. Towards Understanding Smartphone Usage and Sleep with a Crowdsensing Approach. In Adjunct Proceedings of the 2020 ACM International Joint Conference on Pervasive and Ubiquitous Computing and Proceedings of the 2020 ACM International Symposium on Wearable Computers (UbiComp/ISWC '20 Adjunct), September 12-16,2020, Virtual Event, Mexico. ACM, New York, NY, USA, 4 pages. https://doi.org/10.1145/3410530.3414442

\section{INTRODUCTION}

Smartphones are known to cause reliance and even addiction [7]. They are literally taken to bed [1] and used for several purposes from work to leisure, social interactions, and setting wakeup alarms. However, every useful tool can have its negative side effects. Falling asleep with a smartphone is definitely possible, but can the smartphones also keep us awake? In this paper, we present the preliminary results of our study for detecting smartphone usage and sleep quality relationship and draw a pipeline towards using crowdsensed data for analyzing such patterns.

Permission to make digital or hard copies of all or part of this work for personal or classroom use is granted without fee provided that copies are not made or distributed for profit or commercial advantage and that copies bear this notice and the full citation on the first page. Copyrights for components of this work owned by others than ACM must be honored. Abstracting with credit is permitted. To copy otherwise, or republish, to post on servers or to redistribute to lists, requires prior specific permission and/or a fee. Request permissions from permissions@acm.org.

UbiComp/ISWC '20 Adjunct, September 12-16, 2020, Virtual Event, Mexico

(C) 2020 Association for Computing Machinery.

ACM ISBN $\mathrm{xxx}-\mathrm{x}-\mathrm{x} \mathrm{xxx}-\mathrm{x} \mathrm{xxx}-\mathrm{x} / \mathrm{xx} / \mathrm{xx}$

https://doi.org/Xx.Xxxx/Xxxxxx.XXxxxxx
Several physical and psychological studies link smartphone usage, physical exercise, body temperature change, depression, lack of sleep, and general sleepiness during a day together $[4,8,11,13$, 16,18 ] with strong focus on smartphone addiction [2]. Integrally to these works, they follow behavioral habits of young adults or students, limiting the study to a certain age and socioeconomic group. Understandably, college and university students are both native with using smartphones and possibly in a risk of increased stress also causing symptoms of depression, sleepiness, and poor sleep quality. In our work, we aim for more comprehensive demographic picture of the sleep and smartphone usage correlation by involving participants of the different demographic groups by using the crowdsensing approach. As we later discuss, also crowdsensed data collection method has its disadvantages, including issues with the data quality and low control over demographic bias. Thus, based on the mentioned psychological studies [2], sleep quality can be affected by intensive smartphone usage. Interestingly, Katevas et al. [3] show that intense mobile usage alone does not predict severe depression. However, intensive night-time usage of the phone can be seen as a negative predictor for overall wellbeing and sleep quality. One can, of course, rise a question whether the intensive nightly smartphone use is more a symptom or the root cause of lack of sleep.

For most if not all of the sleep quality and smartphone usage studies, the sleep quality data is collected by self-assessment studies. These can be generally accepted among the psychologists and performed by standardized and validated questionnaire forms. For example, Randjelović et al. [15] detected statistically significant correlation between subjective sleep quality and mobile phone usage by using standardized tests. However, actually sensing the sleep quality instead of, or in addition to the self-assessments offers a powerful tool for HCI researchers to see into the nightly behavior patterns of the study participants. Even the smartphone itself can be considered as a sleep sensor [9] with some accuracy. This is the direction we aim, too. However, for this paper, we present the preliminary results by using the PHQ-8 self-assessment questionnaire for depression and especially focus on its questions related sleep and tiredness.

In this paper, we present the first insights of our study to detect behavioral patterns between smartphone usage and sleep quality by using crowdsensed data. To summarize, the contributions of this paper are the following:

- We utilize a large-scale mobile usage dataset and PHQ-8 depression questionnaire answered by 743 participants from varying age groups and socioeconomic backgrounds.

- We suggest there are promising opportunities for future work leading towards understanding daily smartphone usage patterns and sleep quality in the wild, by using crowdsensing approach. 


\section{WELLBEING SELF-ASSESSMENT AND MOBILE USAGE}

The data in this study is collected between 14 March 2018 and 25 August 2018 by using Carat [12], an open source platform for gathering mobile app usage. The Carat app installed in the participants' smartphones collects data consists variable measurements of running apps, timestamp, app state (foreground or background), and participants' anonymous user id. In this study, we obtained the mobile usage data of 743 participants. We focus only on the foreground apps that the users have actively interacted with. From the self-reported demographic data, there are $86 \%$ male and $11 \%$ female. There is a clear bias towards male over female as we cannot control over the aspects like the gender of the participants in this kind of crowdsensed studies. We have $32 \%$ participants from age group $25-34,26 \%$ from age group 45-64, 25\% from 35-44 age group. Other two age groups of our data (18-24 and 65 over) have $12 \%$ and $4 \%$ users.

PHQ-8 is a self-reported assessment where the participants rank eight daily aspects of different feelings such as interest in doing things, trouble falling asleep, and interest in doing things [6]. The participants are asked to consider their feelings on last two weeks when taking the questionnaire. The participants rank items with 0 (Not at all), 1 (Several days), 2 (More than half the days) and 3 (Nearly everyday). In this study, we collected the response of PHQ-8 questionnaire from the 743 participants whose app category usage data we also have from the two-week period before the questionnaire was taken. The PHQ- 8 questionnaire was provided to the Carat platform users through the user questionnaire tool which is implemented as a part of the Android version of Carat. The app usage data and PHQ-8 are mapped by unique and anonymous user id. The apps are categorized by considering Google Play categories such as Communication, Music, and Social. We study hour-based app usage indicating whether an app category was used or not, similarly to the study of mobile usage between different cultures and countries [14].

For understanding the effect of daily app usage over the PHQ-8 answers, we prepare a hourly-based presentation as the following: We study each question individually, dividing participants to corresponding groups based on their answers to each question. For each group $(B)$ we look at their hourly app usage in the last two-week period before responding to the questionnaire, and calculate the fraction $x_{i}$ of users using a given app category for each hour $i$ ( 0 to 23), and construct the vector $X=x_{0}, x_{1}, \ldots, x_{23}$ of these fractions. We also calculate a vector 'all' for usage of certain app category over all the participants $(Y)$. To compare $X$ and $Y$, we calculate the coefficient of determination $\left(R^{2}\right)$ of the linear relationship between $X$ and $Y$ where $X$ is the explanatory/independent and $Y$ is the dependent/criterion variable. High $R^{2}$ indicates that the app usage of people answering certain way to the questionnaire is statistically similar with all participants together. Low $R^{2}$ indicates that people who answered certain way to the questionnaire are using the app statistically differently in comparison to the all participants.

\section{PRELIMINARY RESULTS}

PHQ-8 statement "Little interest or pleasure in doing things" is marked with 0 (Not at all) by 719 users (highest number of user) and marked with 3 (Nearly everyday) by 115 users (lowest number of user) (Figure 1(a)). PHQ-8 statement "Trouble falling or staying asleep, or sleeping too much" is marked with 0 (Not at all) by 655 users and marked with 3 (Nearly everyday) by 127 users (Figure 1(b)). PHQ-8 statement "Feeling tired or having little energy" is marked with 1 (Several days) by 687 users and marked with 3 (Nearly everyday) by 139 users (Figure 1(c)). The results are focused from two viewpoints for popular app categories: (1) hourly app category usage frequency by people and (2) if frequency of participants using particular category for each PHQ-8 answer can explain the overall usage of that same category by all the participants ( $R^{2}$ statistics).

Communication apps: Communication apps are popular among the users as it includes apps like Messaging, chat/IM [17]. A preliminary study of PHQ-8 question "Trouble falling or staying asleep, or sleeping too much" (Q3) can lead us to further research on crowdsensed sleep quality data and the effect of smartphone app usage. Figure 1(e) shows the participant frequency of Communication category usage based on PHQ-8 question Q3 against PHQ-8 answers for each hour. The participant frequency of Communication usage is high among the users who have worsened sleep quality "Nearly every day" and usage frequency is significantly less among the users who "Not at all" or "Several days" have trouble falling asleep or sleep too much. Previous study of Murnane et al. [10] suggests that usage of apps like Communication affects sleep interruptions as well as sleep duration. In their study, the usage of Communication is high in midnight which is similar to our study where we observe people with the PHQ-8 level "Nearly everyday" for worsened sleep quality use Communication apps mostly between 0 to 4 hour. This implicates that Communication usage frequency is high among the people who has worsened sleep quality.

We use the $R^{2}$ measure to see if the frequency of participants using Communication for each PHQ-8 answer can explain the overall Communication usage over worsened sleep quality. For question Q3 and category Communication, the $R^{2}$ fit is lower (0.52) for people who answered "Nearly everyday" than for people who answered "More than half the days" $\left(R^{2}=0.76\right)$, "Several days" $\left(R^{2}=0.95\right)$ and "Not at all" $\left(R^{2}=0.88\right)$. Lower fit indicates that the users of the Communication apps with worsened sleep quality "Nearly everyday" are statistically different from overall Communication usage. The frequency of participants for hourly Communication usage based on PHQ-8 question "Little interest or pleasure in doing things" (Q1) is shown in Figure 1(d) where the frequency of Communication usage is high mostly from 10 to 23 hours and also from 0 to 1 hour among the users who feel "Little interest or pleasure in doing things" "Nearly every day" and "More than half the days". This indicates that participants with little interest in doing things spend more of their time using Communication apps.

Social apps: Social category is popular as it includes social networking apps [17]. In our study, we look at the effects of the Social usage with the sleep quality-related question Q3 ("Trouble falling or staying asleep, or sleeping too much"). Following the similar analysis approach like Communication, Figure 1(g) shows the participant frequency of hourly Social category usage based on Q3 against PHQ-8 answers for each hour. The frequency of Social usage is high among the users who have trouble falling asleep or oversleep "Nearly every day" and usage frequency is significantly less among the users who "Not at all" or "Several days" feel "Trouble falling or staying asleep, or sleeping too much". As per Murnane et al. [10], for Social apps, the highest usage is between $7 \mathrm{pm}$ and midnight. Similarly 
Q1 Little pleasure in doing things

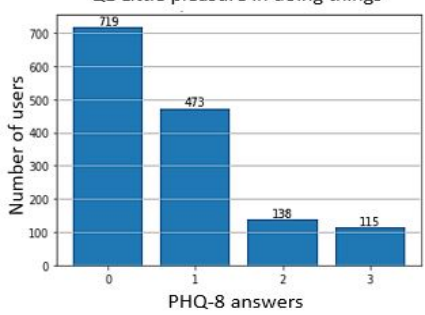

(a)

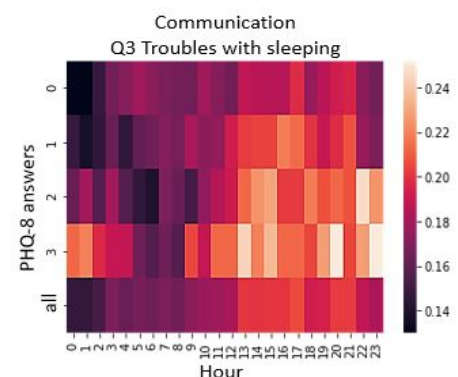

(e)

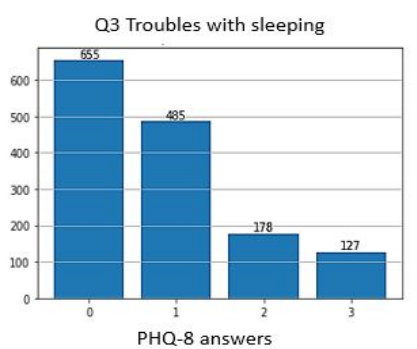

(b)

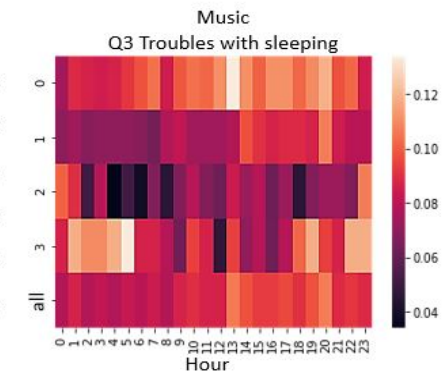

(f)

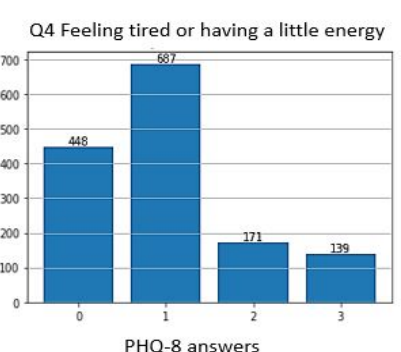

(c)

Social Q3 Troubles with sleeping

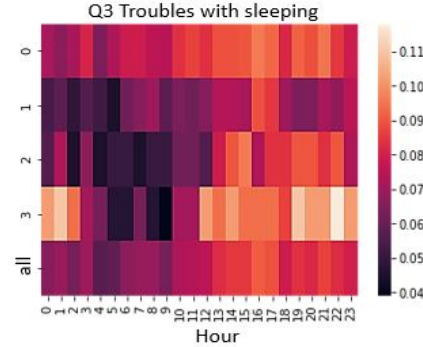

Hour
(g)
Communication Q1 Little pleasure in doing things

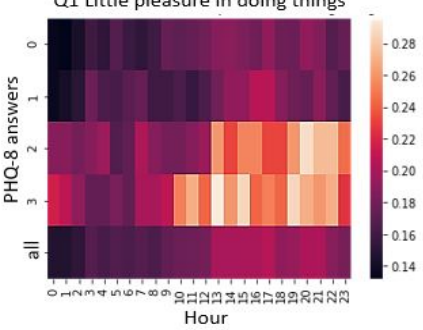

(d)

Music

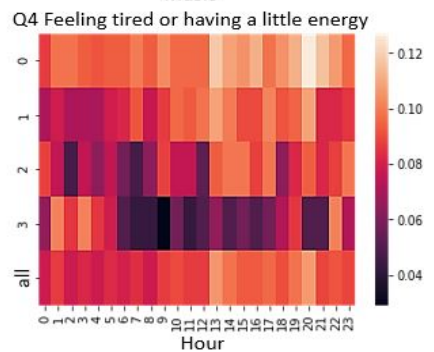

(h)

Figure 1: Distribution of user count for PHQ-8 answers for different PHQ-8 questions (a, b, c). Hourly application category usage frequencies against PHQ-8 answers for different PHQ-8 questions (d, e, f, g, h).

in our study, we see people who answered "Nearly everyday" for worsened sleep quality use Social apps mostly between $7 \mathrm{pm}$ to $2 \mathrm{am}$.

Our study implies that Social category usage is high among the people who has worsened sleep quality. Now if we discuss about the $R^{2}$ measures, we see that the value of $R^{2}$ is lower $(0.65)$ for people who answered "Nearly everyday" to Q3 than for people who answered otherwise ( $R^{2}$ values $\left.0.85-0.91\right)$. Lower fit indicates that those users who most often have problems with sleep quality use Social apps in a statistically different way when compared to all of the users of the Social apps.

Music apps: Music has significant effect on the betterment of anxiety, fatigue and depression [19]. We look for any effect of Music app usage on the PHQ-8 question "Trouble falling or staying asleep, or sleeping too much" (Q3). For Music, the results are quite similar to Communication and Social, the only difference is the usage frequency being also a little high by the people who has good quality sleep during late hours. The reason could be music playing a role to help falling asleep as previous study by Wang et al. [20] analyzed that music therapy improves chronic sleep disorders and worsened sleep quality. Analyzing if the frequency of participants who used Music apps in the given hour for different PHQ-8 answers can explain the overall usage of Music by all the participant for worsened sleep quality, $R^{2}$ has a low value for the answers "Nearly everyday" (0.13) and "More than half the days" (0.33) where other answers have higher fit to all the participants $\left(R^{2}\right.$ is 0.78 and 0.85$)$. Lower fit indicates that the frequency of users for hourly Music app usage for answer "Nearly everyday" and "More than half the days" has a statistically different variance around the mean of the overall usage of that same app category by all the participants.
From Figure 1(h) we can see the frequency of users for the hourly Music apps usage for PHQ-8 question Q4, "Feeling tired or having little energy". The frequency of Music category usage is mostly high during most of the hours of a day (specially during the hours from $1 \mathrm{pm}$ to $10 \mathrm{pm}$ ) among the users who "Not at all" feel "tired or having little energy". The frequency of Music category usage is low during most of the hours of a day (mostly from 6am to $6 \mathrm{pm}, 8 \mathrm{pm}$ to $9 \mathrm{pm}$, and $11 \mathrm{pm}$ to midnight) among the users who feel "tired or having little energy" "Nearly every day". Previous study suggests that Music has significant effect on the betterment of fatigue or tiredness [19].

Comparing our analysis with the study of Tsai et al. [19], we can presume that, people who are "Not at all" suffering from tiredness or lack of energy use Music category during most of the hours of their days. In simpler terms, participants who are energetic spend more of their time using Music apps. We also used the $R^{2}$ values to analyze the relationship between the frequency of participants using Music category in a given hour and the distribution of PHQ-8 answers. For respondents of "Feeling tired or having little energy" (Q4), all PHQ-8 answers have high fit ( $R^{2}$ is between 0.63-0.87) to the Music app usage, except the answer "Nearly everyday" that has a significantly lower fit $\left(R^{2}=0.12\right)$. This means participants who most often feel tired or little energy use Music apps in a significantly different manner than other participants.

Other app categories: Games: Analyzing the effect of Games with PHQ-8 answer levels and sleep quality (Q3), we have only little highlighting results as the usage of Games apps is overall quite low. We see the usage frequency is very high almost all of the hours of a day for PHQ-8 answer "Nearly everyday" and very low among the users for PHQ-8 level "Not at all" and "Several Days". This gives 
little or no insight about the app usage sleep quality of users overall as the users are either not at all or whole day using Games apps. These results differ from previous studies [1] where people were seen using high amount of games on the nightfall. Productivity apps: Even if they are very popular among smartphone users [14], there is only little significant pattern of usage for Productivity apps for worsened sleep quality. Overall the usage is high among the people who answered "Not at all" and "Several Days" than for people who answered "Nearly everyday" for Q3 during most of the hours of a day. This might be due to the Productivity being very board category including apps for various different purposes, such as calendars, notepads, and Google Drive services. Analysis of individual apps instead of the whole category could lead into more meaningful results. Travel and Local apps: There is only little findings for Travel and Local apps for worsened sleep quality which is quite as expected as traveling and sleep may have little or no common. For question "Little interest or pleasure in doing things", interestingly people who answered "Nearly everyday" are the most frequent users of Travel and Local apps for most of the hours of a day. However, with more socioeconomic background (e.g. how much people commute daily) this category could provide more interesting insights.

\section{DISCUSSION AND CONCLUSIONS}

The participants of this study voluntarily allow the Carat platform to report their mobile usage and to take the PHQ-8 questionnaire. Thus we have no control over the demographic factors of the participants like gender or age. In this study, the participants are biased towards male over female. A more demographically balanced participants can provide more gender based insights in addition to our current approach of this study. As our focus was to analyze with a good number of sample, we did not randomly cut the number of male participants' data to bring it equal to the ratio of female participant which would reduce the size of our data drastically. As a preliminary study, our work motivates us to further collect crowdsensed data with demographically balanced participants to avoid the bias of the result towards certain demographic aspects. Our study focuses on hourly app category usage, which can be reduced to minutes or more frequent sampling period to get more detailed view of the participants' interaction with the app category usage.

Self-reported questionnaires like PHQ-8 are widely used by the researchers for studies like depression but nowadays sensor-based data can provide reliable source of getting insight about user activities (i.e. sleep). This leads us to investigate, as a future work, correlation and causality between sleep quality parameters and mobile app behavior by using sensor devices such as Oura - a wearable ring that can track and measure variables related to sleep quality [5]. In this study, we highlight the first results for sleep quality of people based on the PHQ-8 question "Trouble falling or staying asleep, or sleeping too much", which tells about the worsened sleep quality including both "sleep deprivation" and "oversleep". Though PHQ-8 questionnaire is mostly used for depression studies, our work draws attention to the pontential of individual PHQ-8 questions as "early warning" indicators of secondary issues the respondent may be having. For example, if the user is having sleep-related issues, they may be linked to depression but not necessarily caused by it entirely. Our preliminary method of identifying patterns between sleep quality and application use relies on self-reporting, which may result in less accurate and more sparse data; however, the method is widely applicable as it does not require supervision, intervention, or carrying or wearing an extra device. This paper preliminary highlights the impact of app category usage on overall self reported sleep quality which gives us interesting findings for apps like Communication, Social, Music with some potential results. This can lead us to further more interesting insights with crowdsensed data dedicated to collect sleep quality reports of the users.

\section{REFERENCES}

[1] Böhmer, Hecht, Schöning, Krüger, and Bauer. 2011. Falling asleep with Angry Birds, Facebook and Kindle: a large scale study on mobile application usage. In 13th Int. Conf. on Human-Computer Interaction with Mobile Devices and Services. 47-56.

[2] Demirci, Akgönül, and Akpinar. 2015. Relationship of smartphone use severity with sleep quality, depression, and anxiety in university students. J. of Behavioral Addictions 4, 2 (2015), 85-92.

[3] Katevas, Arapakis, and Pielot. 2018. Typical phone use habits: Intense use does not predict negative well-being. In 20th Int. Conf. on Human-Computer Interaction with Mobile Devices and Services. 1-13.

[4] Katsumata, Noda, Isokawa, Katayama, Okoshi, and Nakazawa. 2019. SleepThermo: the affect of in-cloth monitored body temperature change during sleep on human well-being. In ACM Int. Joint Conf. on Pervasive and Ubiquitous Computing and ACM Int. Symp. on Wearable Computers. 1174-1177.

[5] Koskimäki, Kinnunen, Kurppa, and Röning. 2018. How do we sleep: a case study of sleep duration and quality using data from Oura Ring. In Int. Symp. on Pervasive and Ubiquitous Computing and Wearable Computers.

[6] Kroenke, Strine, Spitzer, Williams, Berry, and Mokdad. 2009. The PHQ-8 as a measure of current depression in the general population. J. of Affective Disorders 114, 1-3 (2009), 163-173.

[7] Kwon, Lee, Won, Park, Min, Hahn, Gu, Choi, and Kim. 2013. Development and validation of a smartphone addiction scale (SAS). PloS one 8, 2 (2013).

[8] Liu, Tamminen, Korhonen, Röning, and Riekki. 2019. Prediction of sleep efficiency from big physical exercise data. In ACM Int. Joint Conf. on Pervasive and Ubiquitous Computing and ACM Int. Symp. on Wearable Computers. 1186-1189.

[9] Min, Doryab, Wiese, Amini, Zimmerman, and Hong. 2014. Toss'n'turn: smartphone as sleep and sleep quality detector. In SIGCHI Conf. on Human Factors in Computing Systems. 477-486.

[10] Murnane, Abdullah, Matthews, Kay, Kientz, Choudhury, Gay, and Cosley. 2016. Mobile manifestations of alertness: Connecting biological rhythms with patterns of smartphone app use. In 18th Int. Conf. on Human-Computer Interaction with Mobile Devices and Services. 465-477.

[11] Nowreen and Ahad. 2018. Effect of smartphone usage on quality of sleep in medical students. Nat. J. of Physiology, Pharmacy and Pharmacology 8, 10 (2018).

[12] Oliner, Iyer, Stoica, Lagerspetz, and Tarkoma. 2013. Carat: Collaborative energy diagnosis for mobile devices. In 11th ACM Conf. on Embedded Networked Sensor Systems. 1-14.

[13] Subramani Parasuraman, Yee, Chuon, and Ren. 2017. Smartphone usage and increased risk of mobile phone addiction: A concurrent study. Int. J. of Pharmaceutical Investigation 7, 3 (2017), 125.

[14] Peltonen, Lagerspetz, Hamberg, Mehrotra, Musolesi, Nurmi, and Tarkoma. 2018. The hidden image of mobile apps: geographic, demographic, and cultural factors in mobile usage. In 20th Int. Conf. on Human-Computer Interaction with Mobile Devices and Services. 1-12.

[15] Randjelović, Stojiljković, Radulović, Ilić, Stojanović, and Ilić. 2019. The association of smartphone usage with subjective sleep quality and daytime sleepiness among medical students. Biological Rhythm Research 50, 6 (2019), 857-865.

[16] Rosen, Carrier, Miller, Rokkum, and Ruiz. 2016. Sleeping with technology: cognitive, affective, and technology usage predictors of sleep problems among college students. Sleep health 2, 1 (2016), 49-56.

[17] Sokolova, Perez, and Lemercier. 2017. Android application classification and anomaly detection with graph-based permission patterns. Decision Support Systems 93 (2017), 62-76.

[18] Thomée, Härenstam, and Hagberg. 2011. Mobile phone use and stress, sleep disturbances, and symptoms of depression among young adults-a prospective cohort study. BMC Public Health 11, 1 (2011), 66.

[19] Tsai, Chen, Chung, Liao, Chi, Chang, and Chou. 2014. Effectiveness of music intervention in ameliorating cancer patients' anxiety, depression, pain, and fatigue: A meta-analysis. Cancer Nursing 37, 6 (2014), E35-E50.

[20] Wang, Sun, and Zang. 2014. Music therapy improves sleep quality in acute and chronic sleep disorders: A meta-analysis of 10 randomized studies. Int. J. of Nursing Studies 51, 1 (2014), 51-62. 